\title{
One-loop holographic Weyl anomaly in six dimensions
}

\author{
James T. Liu and Brian McPeak \\ Michigan Center for Theoretical Physics, Randall Laboratory of Physics, \\ The University of Michigan, \\ 450 Church St., Ann Arbor, MI 48109-1040, U.S.A. \\ E-mail: jimliu@umich.edu, bmcpeak@umich.edu
}

AbStract: We compute $\mathcal{O}(1)$ corrections to the holographic Weyl anomaly for sixdimensional $\mathcal{N}=(1,0)$ and $(2,0)$ theories using the functional Schrödinger method that is conjectured to work for supersymmetric theories on Ricci-flat backgrounds. We show that these corrections vanish for long representations of the $\mathcal{N}=(1,0)$ theory, and we obtain an expression for $\delta(c-a)$ for short representations with maximum spin two. We also confirm that the one-loop corrections to the $\mathcal{N}=(2,0) \mathrm{M} 5$-brane theory are equal and opposite to the anomaly for the free tensor multiplet. Finally, we discuss the possibility of extending the results to encompass multiplets with spins greater than two.

Keywords: AdS-CFT Correspondence, Anomalies in Field and String Theories, Conformal Field Theory

ARXIV EPRINT: 1709.02819 


\section{Contents}

1 Introduction 1

1.1 The six-dimensional Weyl anomaly 2

2 The $\mathcal{O}(1)$ contribution to the holographic Weyl anomaly 4

2.1 Heat kernel coefficients 5

$2.2 \mathcal{N}=(1,0)$ theory $\quad 6$

$2.3 \mathcal{N}=(2,0)$ theory 8

$\begin{array}{lll}3 & \text { Discussion } & 10\end{array}$

A Heat kernel for spins up to two $\quad 12$

$\begin{array}{ll}\text { A.1 Conformally coupled scalar } & 12\end{array}$

$\begin{array}{lll}\text { A.2 Weyl fermion } & 13\end{array}$

$\begin{array}{lll}\text { A.3 Vector } & 13\end{array}$

$\begin{array}{lll}\text { A.4 Self-dual three-form } & 14\end{array}$

$\begin{array}{lll}\text { A.5 Gravitino } & 14\end{array}$

$\begin{array}{lll}\text { A.6 Two-form } & 15\end{array}$

$\begin{array}{lll}\text { A.7 Graviton } & 16\end{array}$

$\begin{array}{lr}\text { B Heat kernel for general spins } & 16\end{array}$

$\begin{array}{ll}\text { B.1 Tracing over generators } & 17\end{array}$

$\begin{array}{ll}\text { B.2 Orthogonal tensors } & 18\end{array}$

$\begin{array}{lll}\text { B.3 Dynkin indices } & 18\end{array}$

$\begin{array}{lll}\text { B.4 Results } & 19\end{array}$

\section{Introduction}

The trace anomalies for conformal field theories provide an important set of quantities that characterize the theory. In two dimensions, Cardy's formula [1] demonstrates that the central charge $c$ is a reliable measure of the degrees of freedom. Furthermore, its physical implication can be seen from the Zamolodchikov $c$-theorem [2] which states that an effective $c$ function can be defined that is monotonically decreasing along renormalization group flows to the infrared. While the picture is perhaps the clearest in two dimensions, recent work extending these results to higher dimensional CFTs has further emphasized the importance of trace anomalies in more general situations.

The AdS/CFT correspondence provides an ideal framework for investigating various anomalies, as they may often be reliably computed on both sides of the strong/weak coupling duality. Such calculations can provide a test of the AdS/CFT correspondence and 
can also yield additional insights on strongly coupled CFTs. Here we focus on the conformal anomaly, which measures the change in the partition function that results from a Weyl scaling of the metric, $\delta g_{\mu \nu}=2 \delta \sigma g_{\mu \nu}$. In particular, for a partition function given by

$$
Z=\int \mathcal{D} \phi \exp (-S[\phi])
$$

we define the anomaly $\mathcal{A}$ by

$$
\delta \log Z=-\int d^{d} x \sqrt{\operatorname{det} g} \delta \sigma \mathcal{A} .
$$

From the holographic point of view, the leading order Weyl anomaly can be obtained from the regularized classical action [3]. In the $\mathrm{AdS}_{5} / \mathrm{CFT}_{4}$ case, this gives the familiar result

$$
c=a=\frac{N^{2}}{4} \frac{\pi^{3}}{\operatorname{vol}\left(\Sigma^{5}\right)},
$$

where IIB supergravity has been compactified on $\operatorname{AdS}_{5} \times \Sigma^{5}$. Additional corrections to the leading order expression may arise from higher derivative modifications to the supergravity action as well as from quantum (i.e. loop) effects.

Holographically, the log-divergent part of the one-loop effective action provides an $\mathcal{O}(1)$ correction to the Weyl anomaly coefficients. This was initially computed for the case of $\mathrm{AdS}_{5} \times S^{5}$ in [4-8], where it was observed that the leading order result (1.3) is shifted according to $N^{2} \rightarrow N^{2}-1$, in agreement with expectations for $\mathrm{SU}(N)$ gauge symmetry. More recently, the one-loop computation in $\mathrm{AdS}_{5}$ has been extended to holographic field theories with reduced or even no supersymmetry [9-12].

The one-loop holographic computation is essentially a sum over contributions from all states in the spectrum of single-trace operators. Curiously, when arranged in terms of fourdimensional $\mathcal{N}=1$ superconformal multiplets, the contribution from long multiplets vanish identically. As a result, only short representations contribute to the $\mathcal{O}(1)$ shift in $a$ and $c$. This allows for a close connection between the central charges and the superconformal index which encodes knowledge of the shortened spectrum [13, 14] (see also [15]).

\subsection{The six-dimensional Weyl anomaly}

Here we wish to extend some of the holographic results for the Weyl anomaly coefficients to six dimensions. In general, the anomaly takes the form

$$
(4 \pi)^{3} \mathcal{A}=(4 \pi)^{3}\langle T\rangle=-a E_{6}+\left(c_{1} I_{1}+c_{2} I_{2}+c_{3} I_{3}\right)+D_{\mu} J^{\mu},
$$

where $E_{6}$ refers to the Euler density, $I_{i}$ are Weyl invariants and the final term is a nonuniversal total derivative. At leading order, Einstein gravity on $\mathrm{AdS}_{7}$ gives a relation of the form $[3,16]$

$$
c_{1}=4 c_{2}=-12 c_{3}=96 a \sim \mathcal{O}\left(N^{3}\right),
$$

which is the six-dimensional analog of (1.3). The relation between the $c_{i}$ coefficients arises naturally in the holographic computation, and is further consistent with six-dimensional $(2,0)$ superconformal invariance. 
The most extensively studied $(2,0)$ theory of relevance is that of $N$ coincident M5branes, which is dual to supergravity on $\mathrm{AdS}_{7} \times S^{4}$. Here the conjectured expression for the central charges are [17-19]

$$
a=-\frac{1}{288}\left(4 N^{3}-\frac{9}{4} N-\frac{7}{4}\right), \quad c=-\frac{1}{288}\left(4 N^{3}-3 N-1\right),
$$

where $c_{1}=4 c_{2}=-12 c_{3}=96 c$. The $\mathcal{O}(N)$ terms arise from $R^{4}$ corrections [17], while the $\mathcal{O}(1)$ terms arise at one-loop $[18,19]$. The $\mathcal{O}(1)$ shift $\delta a=7 / 1152$ was computed in [19] by evaluating the one-loop partition function on global (Euclidean) $\operatorname{AdS}_{7}$ with $S^{6}$ boundary. However, the conjectured $\delta c=1 / 288$ has not yet been directly computed, as the most straightforward computation of one-loop determinants involve highly symmetric spaces with conformally flat boundaries. In such cases, the Weyl invariants vanish, so no information is provided about the $c_{i}$ coefficients.

An alternative approach to the computation of $\delta a$ and $\delta c$ was developed in [5-8] based on a functional Schrödinger approach. In this approach, the contribution of each state to the $\mathcal{O}(1)$ shift in the Weyl anomaly takes the form

$$
\delta \mathcal{A}=-\frac{1}{2}\left(\Delta-\frac{d}{2}\right) b_{d}
$$

where $\Delta$ is the conformal dimension and $b_{d}$ is the heat kernel coefficient for the corresponding $\mathrm{AdS}_{d+1}$ field when restricted to the $d$-dimensional boundary. In principle, since the six-dimensional $b_{6}$ coefficient may be computed on a general curved background, this allows for a full determination of not just the $a$ coefficient but the $c_{i}$ 's as well.

It has been argued in [12], however, that the expression (1.7) cannot in general be valid, as the contribution for a single field should have a more complicated dependence on the conformal dimension $\Delta$. This can be seen explicitly in comparison with the expression for $\delta a$ obtained directly from the one-loop determinant on global AdS. Curiously, however, when (1.7) is summed over the states of a complete supermultiplet, the resulting expression appears to be valid on Ricci-flat backgrounds as it passes all consistency checks and has the expected connection to the index $[12,13]$.

Another line of reasoning has been developed to determine the anomaly coefficients directly from the appropriate conformal higher spin operators on the boundary [20,21]. In particular, it is argued that AdS fields with higher dimensions $\Delta$ correspond to boundary fields whose kinetic operators are greater than second order in derivatives. The factorization of these operators on Ricci-flat backgrounds may serve as a justification of the functional Schrödinger method presented in [5-8].

In this paper, we use (1.7) to compute the $\mathcal{O}(1)$ contribution to the holographic Weyl anomaly of $\mathcal{N}=(1,0)$ theories from maximum spin-2 multiplets in the bulk. Since we consider Ricci-flat backgrounds, we only obtain information on $\delta(c-a)$, where $c$ is some linear combination of the Weyl coefficients (and reduces to the $c$ defined above in the $\mathcal{N}=(2,0)$ case $)$. This is similar to the $\mathrm{AdS}_{5} / \mathrm{CFT}_{4}$ case, where $b_{4} \sim \delta(c-a) R_{\mu \nu \rho \sigma}^{2}$ on Ricci-flat backgrounds. As a consistency check, we find that $\delta(c-a)$ vanishes for long representations of $\mathcal{N}=(1,0)$ supersymmetry, as expected. 
While we would ideally want an expression for the anomaly contribution from arbitrary higher-spin multiplets, this would require a better understanding of the heat kernel coefficients $b_{6}$ of higher-spin operators. This was worked out in $[22,23]$ for general spins in four dimensions. However, a similar expression is lacking in six dimensions. Nevertheless, knowledge of $\delta(c-a)$ for spins up to two is sufficient for computing the holographic Weyl anomaly for 11-dimensional supergravity on $\mathrm{AdS}_{7} \times S^{4}$. In this case, we sum the expression for $\delta(c-a)$ over the Kaluza-Klein spectrum and find the expected result $\delta(c-a)=-1 / 384$, in agreement with (1.6) and the original computation of [18].

\section{The $\mathcal{O}(1)$ contribution to the holographic Weyl anomaly}

As indicated above, the six-dimensional Weyl anomaly may be parameterized as

$$
(4 \pi)^{3} \mathcal{A}=-a E_{6}+\left(c_{1} I_{1}+c_{2} I_{2}+c_{3} I_{3}\right)+D_{\mu} J^{\mu},
$$

where $E_{6}=\epsilon_{6} \epsilon_{6} R R R$ is the six-dimensional Euler density, and the Weyl invariants are given by

$$
\begin{aligned}
I_{1} & =C^{a}{ }_{m n}{ }^{b} C^{m}{ }_{p q}{ }^{n} C^{p}{ }_{a b}^{q}, \\
I_{2} & =C^{a b}{ }_{m n} C^{m n}{ }_{p q} C^{p q}{ }_{a b}, \\
I_{3} & =C^{m n p q} \square C_{m n p q}+\cdots .
\end{aligned}
$$

Superconformal invariance imposes additional constraints on the anomaly coefficients $\left\{a, c_{i}\right\}$. In particular, $\mathcal{N}=(1,0)$ supersymmetry requires $c_{1}-2 c_{2}+6 c_{3}=0$, while $\mathcal{N}=(2,0)$ supersymmetry gives an additional constraint $c_{1}-4 c_{2}=0$. This suggests the parametrization

$$
c_{1}=96\left(c+c^{\prime}+c^{\prime \prime}\right), \quad c_{2}=24\left(c-c^{\prime}+c^{\prime \prime}\right), \quad c_{3}=-8\left(c+3 c^{\prime}-3 c^{\prime \prime}\right),
$$

or equivalently

$$
c=\frac{c_{2}-c_{3}}{32}, \quad c^{\prime}=\frac{c_{1}-4 c_{2}}{192}, \quad c^{\prime \prime}=\frac{c_{1}-2 c_{2}+6 c_{3}}{192} .
$$

This is designed so that $c^{\prime \prime}$ vanishes for superconformal theories, and additionally $c^{\prime}$ vanishes when there is extended $\mathcal{N}=(2,0)$ supersymmetry. The coefficient $c$ is chosen to allow for a quantity analogous to $c-a$ in four dimensions, as will become clear below.

The procedure we use to obtain the $\mathcal{O}(1)$ shift in the anomaly for $\mathcal{N}=(1,0)$ theories is to sum the expression (1.7) over complete representations of the corresponding $\operatorname{OSp}\left(8^{*} \mid 2\right)$ supergroup. However, we first start with states in the bosonic $\operatorname{subgroup} \operatorname{OSp}\left(8^{*} \mid 2\right) \supset$ $\mathrm{SO}(2) \times \mathrm{SU}(4) \times \mathrm{SU}(2)_{R}$ labeled by $D\left(\Delta, j_{1}, j_{2}, j_{3}\right)$ along with $R$-symmetry representation $r$. We thus have

$$
\delta \mathcal{A}(\text { rep })=-\frac{1}{2} \sum_{\text {rep }}(\Delta-3) b_{6}\left(j_{1}, j_{2}, j_{3}\right) .
$$

In the following, we first work out the heat kernel coefficients $b_{6}\left(j_{1}, j_{2}, j_{3}\right)$ on a Ricci-flat background, and then perform the sum over complete supermultiplets with maximum spin two. 


\subsection{Heat kernel coefficients}

For an operator $\Delta=-\nabla^{2}-E$ where $E$ is some endomorphism, the six-dimensional SeeleyDeWitt coefficient $b_{6}(\Delta)$ takes the form $[16,24]$

$$
\begin{aligned}
b_{6}(\Delta)= & \frac{1}{(4 \pi)^{3} 7 !} \operatorname{Tr}\left[18 A_{1}+17 A_{2}-2 A_{3}-4 A_{4}+9 A_{5}+28 A_{6}-8 A_{7}+24 A_{8}+12 A_{9}\right. \\
& +\frac{35}{9} A_{10}-\frac{14}{3} A_{11}+\frac{14}{3} A_{12}-\frac{206}{9} A_{13}+\frac{64}{3} A_{14}-\frac{16}{3} A_{15}+\frac{44}{9} A_{16}+\frac{80}{9} A_{17} \\
& +14\left(8 V_{1}+2 V_{2}+12 V_{3}-12 V_{4}+6 V_{5}-4 V_{6}+5 V_{7}+6 V_{8}+60 V_{9}+30 V_{10}\right. \\
& \left.\left.+60 V_{11}+30 V_{12}+10 V_{13}+4 V_{14}+12 V_{15}+30 V_{16}+12 V_{17}+5 V_{18}-2 V_{19}+2 V_{20}\right)\right] .
\end{aligned}
$$

Here the $A_{a}$ 's form a basis of curvature invariants [16, 25], and the $V_{a}$ 's are built from the endomorphism $E$ and the curvature $F_{i j}$ of the connection [16]. In particular, while the coefficients of the $A_{a}$ 's are universal, the $V_{a}$ terms are specific to the representation.

We follow the conventions spelled out in appendix A of [16], which also give explicit expressions for the $A_{a}$ 's and $V_{a}$ 's. However, we are concerned with only the combinations which are non-vanishing on Ricci-flat backgrounds. These are

$$
A_{5}=\left(\nabla_{i} R_{a b c d}\right)^{2}, \quad A_{9}=R_{a b c d} \nabla^{2} R^{a b c d}, \quad A_{16}=R_{a b}{ }^{c d} R_{c d}{ }^{e f} R_{e f}^{a b}, \quad A_{17}=R_{a i b j} R^{m a n b} R_{m}^{i}{ }_{n}^{j} .
$$

The full list of $A_{a}$ 's, and expressions for the $V_{a}$ 's are given in appendix A.

The invariants $E_{6}$ and $I_{1}, I_{2}$, and $I_{3}$ may be written in terms of the basis $A_{a}$ functions. On a Ricci-flat background, they become

$$
E_{6}=32 A_{16}-64 A_{17}, \quad I_{1}=-A_{17}, \quad I_{2}=A_{16}, \quad I_{3}=3 A_{5}+6 A_{9}+2 A_{16}+8 A_{17} .
$$

As these quantities are not all independent, we will be unable to determine the individual central charges $\left\{a, c_{i}\right\}$ using only a Ricci-flat background. Note that we may construct two combinations that are total derivatives

$$
\begin{aligned}
& D_{1}=\nabla_{a}\left(R_{m n i j} \nabla_{a} R_{m n i j}\right)=A_{5}+A_{9}, \\
& D_{2}=2 \nabla_{a}\left(R_{m n i j} \nabla_{m} R_{a n i j}\right)=-A_{5}+A_{16}+4 A_{17} .
\end{aligned}
$$

This allows us to rewrite (2.8) in terms of the two invariants $A_{16}$ and $A_{17}$

$$
E_{6}=32 A_{16}-64 A_{17}, \quad I_{1}=-A_{17}, \quad I_{2}=A_{16}, \quad I_{3}=-A_{16}-4 A_{17}+6 D_{1}-3 D_{2} .
$$

On a Ricci-flat background, we have the relations $E_{6}=32\left(2 I_{1}+I_{2}\right)$ and $I_{3}=4 I_{1}-I_{2}$ up to a total derivative. As a result, the six-dimensional anomaly, (2.1), takes the form

$$
(4 \pi)^{3} \mathcal{A}=32(c-a) A_{16}-64\left(c-a+3 c^{\prime \prime}\right) A_{17}+D_{\mu} J^{\mu},
$$

on Ricci-flat backgrounds. The implication of this expression is that we will only be able to obtain information on the $\mathcal{O}(1)$ contribution to $c-a$ and to $c^{\prime \prime}$. Since the latter must vanish for superconformal theories, it will serve as a consistency check of our approach. 


\begin{tabular}{|c|c|c|c|c|c|c|c|}
\hline Field & SU(4) Rep & $c_{5}$ & $c_{9}$ & $c_{16}$ & $c_{17}$ & $\gamma_{16}$ & $\gamma_{17}$ \\
\hline$\phi$ & $(0,0,0)=\mathbf{1}$ & 9 & 12 & $44 / 9$ & $80 / 9$ & $17 / 9$ & $-28 / 9$ \\
\hline$\psi$ & $(1,0,0)=\mathbf{4}$ & -20 & -36 & $-202 / 9$ & $-436 / 9$ & $-58 / 9$ & $140 / 9$ \\
\hline$A_{\mu}$ & $(0,1,0)=\mathbf{6}$ & -58 & -96 & $-164 / 3$ & $-344 / 3$ & $-50 / 3$ & $112 / 3$ \\
\hline$C_{\mu \nu \rho}^{+}$ & $(2,0,0)=\mathbf{1 0}$ & 174 & 456 & $-5608 / 9$ & $26504 / 9$ & $-8146 / 9$ & $16352 / 9$ \\
\hline$\Psi_{\mu}$ & $(1,1,0)=\overline{\mathbf{2 0}}$ & 292 & 828 & $3526 / 9$ & $22012 / 9$ & $-1298 / 9$ & $2716 / 9$ \\
\hline$B_{\mu \nu}$ & $(1,0,1)=\mathbf{1 5}$ & 107 & 348 & $2992 / 3$ & $-1616 / 3$ & $2269 / 3$ & $-4508 / 3$ \\
\hline$G_{\mu \nu}$ & $(0,2,0)=\mathbf{2 0}$ & 544 & 1416 & $-1388 / 9$ & $49984 / 9$ & $-9236 / 9$ & $18592 / 9$ \\
\hline
\end{tabular}

Table 1. Heat kernel coefficients $(4 \pi)^{3} 7 ! b_{6}=c_{5} A_{5}+c_{9} A_{9}+c_{16} A_{16}+c_{17} A_{17}$ for fields of spins up to two on a Ricci-flat background. In the last two columns, we tabulate $\gamma_{16}$ and $\gamma_{17}$, where $(4 \pi)^{3} 7 ! b_{6}=\gamma_{16} A_{16}+\gamma_{17} A_{17}+D_{\mu} J^{\mu}$.

This leaves us with a holographic determination of $\delta(c-a)$, which may be combined with the result of [19] for the $\delta a$ coefficient to extract both $\delta c$ and $\delta a$. This, in principle, provides a complete determination of the $\mathcal{O}(1)$ shift in the holographic Weyl anomaly of $\mathcal{N}=(2,0)$ theories. Unfortunately the additional anomaly coefficient $\delta c^{\prime}$ for $\mathcal{N}=(1,0)$ theories cannot be determined in this manner on Ricci-flat backgrounds.

Ideally, we would like to have an expression for the heat kernel coefficient $b_{6}(\Delta)$ for fields transforming in an arbitrary $\left(j_{1}, j_{2}, j_{3}\right)$ representation of the six-dimensional SU(4) Euclidean rotation group. However, this requires understanding of arbitrary higher-spin Laplacians which currently eludes us. There is also some potential ambiguity in relating 'on-shell' states in $\mathrm{AdS}_{7}$ to their corresponding boundary Laplacians in the functional Schrodinger approach of [5]. We thus restrict to spins up to two. The relevant $b_{6}$ coefficients evaluated on a Ricci-flat background are summarized in table 1. The coefficients for $\phi, \psi$, $A_{\mu}$ and $B_{\mu \nu}$ were computed in [16], while the remaining ones are worked out in appendix A.

\section{$2.2 \mathcal{N}=(1,0)$ theory}

We now turn to the superconformal theories, starting with the $\mathcal{N}=(1,0)$ theory. We expect that the anomaly vanishes when summed over long representations, and we will see that this is indeed the case. The $\mathcal{N}=(1,0)$ superconformal algebra is $\operatorname{OSp}\left(8^{*} \mid 2\right)$, with bosonic subgroup $\mathrm{SO}(2,6) \times \mathrm{SU}(2)_{R}$. Here $\mathrm{SO}(2,6)$ is either the isometry group of $\mathrm{AdS}_{7}$ or the six-dimensional conformal group. We label representations of $\operatorname{OSp}\left(8^{*} \mid 2\right) \supset$ $\mathrm{SO}(2,6) \times \mathrm{SU}(2)_{R} \supset \mathrm{SO}(2) \times \mathrm{SU}(4) \times \mathrm{SU}(2)_{R}$ by conformal dimension $\Delta, \mathrm{SU}(4)$ Dynkin labels $\left(j_{1}, j_{2}, j_{3}\right)$ and $\mathrm{SU}(2)_{R}$ Dynkin label $k$ (so that $\mathrm{SU}(2)$ 'spin' is given by $k / 2$ ).

Unitary irreducible representations of the $\mathcal{N}=(1,0)$ theory have been studied and explicitly constructed in [26-30]. The theory has one regular and three isolated short 


\begin{tabular}{|c|c|c|c|c|c|c|}
\hline Level & $\mathrm{SU}(4)$ & $\begin{array}{c}D[0,0,0 ; k] \\
\Delta=2 k\end{array}$ & $\begin{array}{l}C[0,0,0 ; k] \\
\Delta=2 k+2\end{array}$ & $\begin{array}{l}B[0,0,0 ; k] \\
\Delta=2 k+4\end{array}$ & $\begin{array}{l}A[0,0,0 ; k] \\
\Delta=2 k+6\end{array}$ & $\begin{array}{c}L[0,0,0 ; k] \\
\Delta>2 k+6\end{array}$ \\
\hline$\Delta$ & 1 & $k$ & $k$ & $k$ & $k$ & $k$ \\
\hline$\Delta+\frac{1}{2}$ & 4 & $k-1$ & $k-1, k+1$ & $k-1, k+1$ & $k-1, k+1$ & $k-1, k+1$ \\
\hline$\Delta+1$ & $\begin{array}{c}10 \\
6\end{array}$ & $k-2$ & $\begin{array}{c}k \\
k-2, k\end{array}$ & $\begin{array}{c}k \\
k-2, k, k+2\end{array}$ & $\begin{array}{c}k \\
k-2, k, k+2\end{array}$ & $\begin{array}{c}k \\
k-2, k, k+2\end{array}$ \\
\hline$\Delta+\frac{3}{2}$ & $\begin{array}{l}\overline{20} \\
\overline{4}\end{array}$ & $k-3$ & $\begin{array}{c}k-1 \\
k-3, k-1\end{array}$ & $\begin{array}{c}k-1, k+1 \\
k-3, k-1, k+1\end{array}$ & $\begin{array}{c}k-1, k+1 \\
k-3, k-1, k+1, k+3\end{array}$ & $\begin{array}{c}k-1, k+1 \\
k-3, k-1, k+1, k+3\end{array}$ \\
\hline$\Delta+2$ & $\begin{array}{c}20^{\prime} \\
15 \\
1\end{array}$ & $k-4$ & $\begin{array}{c}k-2 \\
k-4, k-2\end{array}$ & $\begin{array}{c}k \\
k-2, k \\
k-4, k-2, k\end{array}$ & $\begin{array}{c}k \\
k-2, k, k+2 \\
k-4, k-2, k, k+2\end{array}$ & $\begin{array}{c}k \\
k-2, k, k+2 \\
k-4, k-2, k, k+2, k+4\end{array}$ \\
\hline$\Delta+\frac{5}{2}$ & $\begin{array}{c}20 \\
4 \\
\end{array}$ & & $k-3$ & $\begin{array}{c}k-1 \\
k-3, k-1 \\
\end{array}$ & $\begin{array}{c}k-1, k+1 \\
k-3, k-1, k+1 \\
\end{array}$ & $\begin{array}{c}k-1, k+1 \\
k-3, k-1, k+1, k+3\end{array}$ \\
\hline$\Delta+3$ & $\begin{array}{c}\overline{10} \\
6\end{array}$ & & & $k-2$ & $\begin{array}{c}k \\
k-2, k\end{array}$ & $\begin{array}{c}k \\
k-2, k, k+2\end{array}$ \\
\hline$\Delta+\frac{7}{2}$ & $\overline{4}$ & & & & $k-1$ & $k-1, k+1$ \\
\hline$\Delta+4$ & 1 & & & & & $k$ \\
\hline Anomaly & $\begin{array}{c}2^{5} \cdot 6 ! \delta(c-a) \\
\delta c^{\prime \prime}\end{array}$ & $\begin{array}{l}1 \\
0\end{array}$ & $\begin{array}{c}57+180 k \\
0\end{array}$ & $\begin{array}{c}303+180 k \\
0\end{array}$ & $\begin{array}{c}-1 \\
0\end{array}$ & $\begin{array}{l}0 \\
0\end{array}$ \\
\hline
\end{tabular}

Table 2. The $\mathcal{N}=(1,0)$ multiplets with maximum spin two, and corresponding holographic Weyl anomaly coefficients $\delta(c-a)$ and $\delta c^{\prime \prime}$. Here $k$ is the $\mathrm{SU}(2)_{R}$ Dynkin label (with spin $\left.=k / 2\right)$. The shortening conditions correspond to those of (2.12), while the last column is the maximum spin-two long representation.

representations, given generically by

$$
\begin{array}{ll}
A\left[j_{1}, j_{2}, j_{3} ; k\right]: & \Delta=\frac{1}{2}\left(j_{1}+2 j_{2}+3 j_{3}\right)+2 k+6, \\
B\left[j_{1}, j_{2}, 0 ; k\right]: & \Delta=\frac{1}{2}\left(j_{1}+2 j_{2}\right)+2 k+4, \\
C\left[j_{1}, 0,0 ; k\right]: & \Delta=\frac{1}{2} j_{1}+2 k+2, \\
D[0,0,0 ; k]: & \Delta=2 k .
\end{array}
$$

For maximum spin two, however, we must restrict to $j_{1}=j_{2}=j_{3}=0$. In this case, it is a simple exercise to perform the sum (2.5) over the multiplet using the values of $\gamma_{16}$ and $\gamma_{17}$ given in table 1 . Comparison with (2.11) then allows us to extract $\delta(c-a)$ and $\delta c^{\prime \prime}$. The results are summarized in table 2.

As a consistency check, we note that the anomaly coefficient $c^{\prime \prime}$ vanishes identically after summation over a complete multiplet. This is a requirement of supersymmetry, but is not manifest from the individual $b_{6}$ coefficients in table 1 . We also see that the anomaly vanishes for the long representation, which agrees with expectations from the $\mathrm{AdS}_{5}$ case $[9,12]$. As for the non-vanishing contributions, note that $\delta(c-a)$ for the $A$ and $D$ type multiplets are equal and opposite. This must be the case, as $A[0,0,0 ; k]$ and $D[0,0,0 ; k+2]$ are "mirror shorts" that sum to become a long multiplet. 


\begin{tabular}{|c|c|c|c|}
\hline Multiplet & $\Delta$ & $2^{5} \cdot 6 ! \delta a$ & $2^{5} \cdot 6 ! \delta(c-a)$ \\
\hline$L[0,0,0 ; k]$ & $>2 k+6$ & 0 & 0 \\
\hline$A[0,0,0 ; k]$ & $2 k+6$ & $10 \Delta^{2}\left(\Delta^{2}-2\right)+\frac{11}{3}$ & -1 \\
\hline$B[0,0,0 ; k]$ & $2 k+4$ & $-10\left(\Delta-\frac{2}{3}\right)^{2}\left(3\left(\Delta-\frac{2}{3}\right)^{2}-14\right)-\frac{530}{9}\left(\Delta-\frac{2}{3}\right)-\frac{419}{9}$ & $90\left(\Delta-\frac{2}{3}\right)+3$ \\
\hline$C[0,0,0 ; k]$ & $2 k+2$ & $10\left(\Delta-\frac{4}{3}\right)^{2}\left(3\left(\Delta-\frac{4}{3}\right)^{2}-14\right)-\frac{530}{9}\left(\Delta-\frac{4}{3}\right)+\frac{419}{9}$ & $90\left(\Delta-\frac{4}{3}\right)-3$ \\
\hline$D[0,0,0 ; k]$ & $2 k$ & $-10(\Delta-2)^{2}\left((\Delta-2)^{2}-2\right)-\frac{11}{3}$ & 1 \\
\hline
\end{tabular}

Table 3. Contribution to the Weyl anomaly coefficients $\delta a$ and $\delta c$ from maximum spin two multiplets for the $\mathcal{N}=(1,0)$ theory. Here $c$ is related to the conventional anomaly coefficients $c_{i}$ according to (2.4). The $\delta a$ coefficient is computed using the results of [19].

Finally, recall that the $\mathcal{N}=(1,0)$ theory admits three independent anomaly coefficients, which we have parametrized as $a, c$ and $c^{\prime}$. Since we only consider Ricci-flat backgrounds, we have only been able to determine the difference $\delta(c-a)$. This may be combined with the holographic $\delta a$ coefficient obtained in [19] to separate out the contributions to $\delta a$ and $\delta c$. These results are presented in table 3. However, we are unable to determine $\delta c^{\prime}$ unless we can move away from Ricci-flat backgrounds.

\section{$2.3 \mathcal{N}=(2,0)$ theory}

We may perform the same computation for the $\mathcal{N}=(2,0)$ theory, noting however that only the $1 / 2$-BPS multiplets have spins less than or equal to two. In this case, the superconformal algebra decomposes as $\mathrm{OSp}\left(8^{*} \mid 4\right) \supset \mathrm{SO}(2,6) \times \mathrm{Sp}(4)_{R} \supset \mathrm{SO}(2) \times \mathrm{SU}(4) \times \mathrm{Sp}(4)_{R}$. The shortening conditions follow the same pattern as (2.12), however with extended $R$ symmetry [26-30]

$$
\begin{array}{lll}
A\left[j_{1}, j_{2}, j_{3} ; k_{1}, k_{2}\right]: & \Delta=\frac{1}{2}\left(j_{1}+2 j_{2}+3 j_{3}\right)+2\left(k_{1}+k_{2}\right)+6, \\
B\left[j_{1}, j_{2}, 0 ; k_{1}, k_{2}\right]: & \Delta=\frac{1}{2}\left(j_{1}+2 j_{2}\right)+2\left(k_{1}+k_{2}\right)+4, \\
C\left[j_{1}, 0,0 ; k_{1}, k_{2}\right]: & \Delta=\frac{1}{2} j_{1}+2\left(k_{1}+k_{2}\right)+2, \\
D\left[0,0,0 ; k_{1}, k_{2}\right]: & \Delta=2\left(k_{1}+k_{2}\right) .
\end{array}
$$

Here $\left(k_{1}, k_{2}\right)$ are Dynkin labels for $\operatorname{Sp}(4)$, with $(1,0)$ denoting the 4 and $(0,1)$ denoting the 5. For maximum spin two, we restrict to the $1 / 2$-BPS multiplets $D[0,0,0 ; 0, k]$ with $\Delta=2 k$. (The case $k=1$ is the free tensor multiplet, while $k=2$ is the stress tensor multiplet.)

The holographic computation of $\delta(c-a)$ and $\delta c^{\prime \prime}$ for the $D[0,0,0 ; 0, k]$ multiplets are shown in table 4 . The case $k \geq 4$ is generic, and we do not include $k=1$, which is a supersingleton and would not appear in a holographic computation. The special case $k=3$ fits into the generic pattern, and in fact so does $k=2$, although it requires separate treatment because of the presence of massless modes. For $k=2$, the states in $D[0,0,0 ; 0,2]$ 


\begin{tabular}{|c|c|c|c|c|}
\hline$\Delta$ & $\mathrm{SU}(4)$ & $D[0,0,0 ; 0,2]$ & $D[0,0,0 ; 0,3]$ & $D[0,0,0 ; 0, k \geq 4]$ \\
\hline $2 k$ & $\mathbf{1}$ & $(0,2)$ & $(0,3)$ & $(0, k)$ \\
\hline $2 k+\frac{1}{2}$ & $\mathbf{4}$ & $(1,1)$ & $(1,2)$ & $(1, k-1)$ \\
\hline $2 k+1$ & $\mathbf{6}$ & $(2,0)$ & $(2,1)$ & $(2, k-2)$ \\
& $\mathbf{1 0}$ & $(0,1)$ & $(0,2)$ & $(0, k-1)$ \\
\hline $2 k+\frac{3}{2}$ & $\overline{\mathbf{2 0}}$ & $(1,0)$ & $(1,1)$ & $(1, k-2)$ \\
& $\overline{\mathbf{4}}$ & & $(3,0)$ & $(3, k-3)$ \\
\hline $2 k+2$ & $\mathbf{2 0}$ & $(0,0)$ & $(0,1)$ & $(0, k-2)$ \\
& $\mathbf{1 5}$ & & $(2,0)$ & $(2, k-3)$ \\
& $\mathbf{1}$ & & & $(4, k-4)$ \\
\hline $2 k+\frac{5}{2}$ & $\mathbf{2 0}$ & & $(1,0)$ & $(1, k-3)$ \\
& $\mathbf{4}$ & & $(0,0)$ & $(0, k-3)$ \\
\hline $2 k+3$ & $\overline{\mathbf{1 0}}$ & & & $(2, k-4)$ \\
& $\mathbf{6}$ & & & $(1, k-4)$ \\
\hline $2 k+\frac{7}{2}$ & $\overline{\mathbf{4}}$ & & & $6 k(k-1)+1$ \\
\hline $2 k+4$ & $\mathbf{1}$ & & 0 & 0 \\
\hline Anomaly & $384 \delta(c-a)$ & 13 & & \\
& $\delta c^{\prime \prime}$ & 0 & & \\
\hline
\end{tabular}

Table 4. The $\mathcal{N}=(2,0) 1 / 2$-BPS (maximum spin two) representation $D[0,0,0 ; 0, k]$ and corresponding holographic Weyl anomaly coefficients $\delta(c-a)$ and $\delta c^{\prime \prime}$. Entries are $\operatorname{Sp}(4)_{R}$ representations specified by Dynkin labels $\left(k_{1}, k_{2}\right)$.

are

$$
\begin{aligned}
& D(4 ; 0,0,0)_{\mathbf{1 4}}+D\left(4 \frac{1}{2} ; 1,0,0\right)_{\mathbf{1 6}}+D(5 ; 0,1,0)_{\mathbf{1 0}} \\
& +D(5 ; 2,0,0)_{\mathbf{5}}+D\left(5 \frac{1}{2} ; 1,1,0\right)_{\mathbf{4}}+D(6 ; 0,2,0)_{\mathbf{1}}
\end{aligned}
$$

where $D\left(\Delta ; j_{1}, j_{2}, j_{3}\right)$ labels the $\mathrm{SO}(2,6)$ representation and the subscript labels the $\operatorname{Sp}(4)_{R}$ representation. The massless vector, gravitino and graviton representations can be obtained from the corresponding massive representations by subtracting out null states according to

$$
\begin{aligned}
D(5 ; 0,1,0) & =D(5+\epsilon ; 0,1,0)-D(6 ; 0,0,0), \\
D\left(5 \frac{1}{2} ; 1,1,0\right) & =D\left(5 \frac{1}{2}+\epsilon ; 1,1,0\right)-D\left(6 \frac{1}{2} ; 1,0,0\right), \\
D(6 ; 0,2,0) & =D(6+\epsilon ; 0,2,0)-D(7 ; 0,1,0) .
\end{aligned}
$$

(Note that the three-form, $D(5 ; 2,0,0)$, is massive, so no subtraction is required.) Taking these null states into account then gives the result $\delta(c-a)=13 / 384$ for $k=2$ shown in table 4 . 
Although $k=2$ and $k=3$ are special cases, the holographic anomaly coefficient $\delta(c-$ $a)=(1 / 384)(6 k(k-1)+1)$ is in fact universal. Combining this with $\delta a=-(7 / 1152)(6 k(k-$ $1)+1)$ obtained in [19] then allows us to separate out the individual coefficients

$$
D[0,0,0 ; 0, k \geq 2]: \quad \delta a=-\frac{1}{288} \cdot \frac{7}{4}(6 k(k-1)+1), \quad \delta c=-\frac{1}{288}(6 k(k-1)+1) .
$$

As an application, consider the $\mathcal{N}=(2,0)$ theory obtained by compactifying 11dimensional supergravity on $\mathrm{AdS}_{7} \times S^{4}$. The Kaluza-Klein spectrum is simply

$$
\oplus_{k \geq 2} D[0,0,0 ; 0, k]
$$

where $k=2$ corresponds to the 'massless' supergravity sector. The anomaly coefficients $\delta a$ and $\delta c$ may be computed by summing over the Kaluza-Klein levels

$$
\delta a=-\frac{1}{288} \cdot \frac{7}{4} \sum_{k=2}^{\infty}(6 k(k-1)+1), \quad \delta c=-\frac{1}{288} \sum_{k=2}^{\infty}(6 k(k-1)+1) .
$$

Following [19], we regulate the sums using a hard cutoff. This amounts to setting $\sum_{k=1}^{\infty} k^{n}=0$ for any $n \geq 0$. This implies $\sum_{k=2}^{\infty} f(k)=-f(1)$, where $f(k)$ is polynomial in $k$. As a result, the regulated anomaly for $\operatorname{AdS}_{7} \times S^{4}$ is

$$
\delta a=\frac{1}{288} \cdot \frac{7}{4}, \quad \delta c=\frac{1}{288} .
$$

This is equal and opposite to the result for the conformal anomaly of the free tensor multiplet computed in [17], and agrees with the $\mathcal{O}(1)$ contributions in (1.6).

\section{Discussion}

While the six-dimensional Weyl anomaly coefficients are conventionally parametrized as $a$, $c_{1}, c_{2}$ and $c_{3}$, we have found it convenient to use an alternate linear combination of the $c_{i}$ 's given in (2.4). Holographically, the leading order anomaly coefficients (assuming Einstein gravity in the bulk) satisfy the relation

$$
c=a, \quad c^{\prime}=0, \quad c^{\prime \prime}=0 .
$$

(While $c^{\prime \prime}=0$ must hold for superconformal theories, this holographic result is independent of whether the theory is supersymmetric or not.) At the one-loop level, we have been able to extend this leading order result by computing $\delta(c-a)$ using the expression (1.7) obtained through a functional Schrödinger method [5-8].

It is reasonable to question whether the use of (1.7) is valid, as it disagrees with the direct computation of $\delta a$ performed in $[12,19]$. A quick way to see this is to note that $\delta a$ in table 3 is a fourth order polynomial in $\Delta$, while the result of summing (1.7) over a supermultiplet can be at most quadratic in $\Delta$. (One power comes directly from (1.7), while another can arise from the dimension of the shortened representation.) If $\delta(c-a)$ was expected to be cubic or higher in $\Delta$, then our result, as shown in the last column of 
table 3, cannot possibly be correct. However, we now demonstrate that $c-a$ can be at most linear in $\Delta$, which is consistent with application of (1.7).

To see this, recall that, in superconformal field theories, the stress tensor is contained in a multiplet of currents, so that there is a corresponding multiplet of anomalies. For $\mathcal{N}=(1,0)$ theory, the " $t$ Hooft anomalies are characterized by the anomaly polynomial

$$
\mathcal{I}_{8}=\frac{1}{4 !}\left[\alpha c_{2}(R)^{2}+\beta c_{2}(R) p_{1}(T)+\gamma p_{1}(T)^{2}+\delta p_{2}(T)\right]
$$

and the relation to the Weyl anomaly coefficients has recently been worked out [20,31-33]

$$
a=-\frac{1}{72}\left(\alpha-\beta+\gamma+\frac{3}{8} \delta\right), \quad c-a=-\frac{\delta}{192}, \quad c^{\prime}=\frac{1}{432}\left(\beta-2 \gamma+\frac{1}{2} \delta\right) .
$$

Since $\alpha$ is the coefficient of the $\left[\mathrm{SU}(2)_{R}\right]^{4}$ anomaly, it can be at most fifth power in $\Delta$, where the extra power comes from the dimension of the representation. Similarly, $\beta$ can be at most cubic in $\Delta$, while $\gamma$ and $\delta$ can be at most linear in $\Delta$. This in turn demonstrates that $a$ will be at most fifth power in $\Delta, c^{\prime}$ will be at most cubic and $c-a$ will be at most linear. Thus the functional Schrödinger method is indeed compatible with $\delta(c-a)$. However, we also see this approach cannot be used to compute either $\delta a$ alone or $\delta c^{\prime}$. Thus, while it would be desirable to compute $\delta c^{\prime}$ in these theories, we do not expect that it can be done using this approach.

While we have focused on short multiplets with spins $\leq 2$, it would be desirable to work more generally with higher-spin multiplets. To do so, we would need knowledge of the $b_{6}$ coefficients for arbitrary spin fields. This in turn depends on the form of the higher-spin Laplacian. In general, this depends on the bulk dynamics of the higher-spin field and the further restriction to the boundary following from the procedure of [5-8]. For higher-spin bosons, it is natural to take a bulk Laplacian of the form $\Delta=-\square-E$ with the endomorphism $E=\Sigma_{a b} R^{a b c d} \Sigma_{c d}$, where $\Sigma_{a b}$ are $\mathrm{SU}(4)$ generators in the appropriate bosonic higher-spin representation. However, the situation is less clear for fermions. The natural generalization would be to simply take $\Sigma_{a b}$ to be in a fermionic higher-spin representation. However, this does not agree with the square of the Dirac operator for ordinary spin- $1 / 2$ fermions. Nevertheless, it is possible that the use of a universal endomorphism term for bosons and fermions would be appropriate when tracing over supermultiplets. Along these lines, we have computed the $b_{6}$ coefficient for general higher-spin representations in appendix B.

Finally, part of our motivation for exploring the $\mathcal{O}(1)$ contributions to the holographic Weyl anomaly is to make a connection to the $\mathcal{N}=(1,0)$ superconformal index. As in the $\mathrm{AdS}_{5} / \mathrm{CFT}_{4}$ case, the bulk one-loop corrections to the Weyl anomaly vanish for long representations, so it is natural to expect that these corrections can be obtained from the index. More generally, we anticipate that the one-loop matching between $\delta(c-a)$ and the index can be extended to the full set of anomaly coefficients $a$ and $c_{i}$, even in theories without holographic duals. This would then open up a new path towards characterizing superconformal field theories in six dimensions.

\section{Acknowledgments}

We wish to thank A. Arabi Ardehali, F. Larsen and P. Szepietowski for stimulating discussions. This work was supported in part by the US Department of Energy under Grant 
No. DE-SC0007859. The work of B. McPeak was partially supported by a Rackham 2016 Spring/Summer Research Grant.

\section{A Heat kernel for spins up to two}

The Seeley-DeWitt coefficients $b_{n}(\Delta)$ depend on the field and the form of the second order operator $\Delta$. In four dimensions, the appropriate operators for irreducible fields up to spin two are listed in [23]. Here we write down the analogous operators in six dimensions and compute the contribution of each to the anomaly.

We start with the basis of curvature invariants $[16,25]$

$$
\begin{aligned}
A_{1} & =\square^{2} R, \quad A_{2}=\left(\nabla_{a} R\right)^{2}, \quad A_{3}=\left(\nabla_{a} R_{m n}\right)^{2}, \quad A_{4}=\nabla_{a} R_{b m} \nabla^{b} R^{a m}, \quad A_{5}=\left(\nabla_{a} R_{m n i j}\right)^{2}, \\
A_{6} & =R \square R, \quad A_{7}=R_{a b} \square R^{a b}, \quad A_{8}=R_{a b} \nabla_{m} \nabla^{b} R^{a m}, \quad A_{9}=R_{a b m n} \square R^{a b m n}, \quad A_{10}=R^{3} \\
A_{11} & =R R_{a b}^{2}, \quad A_{12}=R R_{a b m n}^{2}, \quad A_{13}=R_{a}^{m} R_{m}^{i} R_{i}^{a}, \quad A_{14}=R_{a b} R_{m n} R^{a m b n}, \\
A_{15} & =R_{a b} R^{a m n l} R_{m n l}^{b}, \quad A_{16}=R_{a b}{ }^{c d} R_{c d}{ }^{e f} R_{e f}^{a b}, \quad A_{17}=R_{a i b j} R^{m a n b} R_{m}^{i}{ }^{j}{ }_{n} .
\end{aligned}
$$

The $b_{6}$ coefficient may be computed from the expression (2.6), where the $V_{a}$ 's are given by

$$
\begin{aligned}
V_{1} & =\nabla_{k} F_{i j} \nabla^{k} F^{i j}, \quad V_{2}=\nabla_{j} F_{i j} \nabla^{k} F^{i k}, \quad V_{3}=F_{i j} \square F^{i j}, \quad V_{4}=F_{i j} F^{j k} F_{k}^{i}, \\
V_{5} & =R_{m n i j} F^{m n} F^{i j}, \quad V_{6}=R_{j k} F^{j n} F_{n}^{k}, \quad V_{7}=R F_{i j} F^{i j}, \quad V_{8}=\square^{2} E, \quad V_{9}=E \square E, \\
V_{10} & =\nabla_{k} E \nabla^{k} E, \quad V_{11}=E^{3}, \quad V_{12}=E F_{i j}^{2}, \quad V_{13}=R \square E, \quad V_{14}=R_{i j} \nabla^{i} \nabla^{j} E, \\
V_{15} & =\nabla_{k} R \nabla^{k} E, \quad V_{16}=E^{2} R, \quad V_{17}=E \square R, \quad V_{18}=E R^{2}, \\
V_{19} & =E R_{i j}^{2}, \quad V_{20}=E R_{i j k l}^{2} .
\end{aligned}
$$

Here $\Delta=-\nabla^{2}-E$ and $F_{i j}$ is the curvature of the connection, $\left[\nabla_{i}, \nabla_{j}\right]=F_{i j}$. Below we present the $V$-terms for each field after tracing over the representation.

\section{A.1 Conformally coupled scalar}

The conformally coupled scalar has $E=-\frac{1}{5} R$ and $F_{i j}=0$, so the $V$-terms are:

\begin{tabular}{|c|c|c|c|c|c|c|c|c|c|}
\hline$V_{1}$ & $V_{2}$ & $V_{3}$ & $V_{4}$ & $V_{5}$ & $V_{6}$ & $V_{7}$ & $V_{8}$ & $V_{9}$ & $V_{10}$ \\
\hline 0 & 0 & 0 & 0 & 0 & 0 & 0 & $-\frac{1}{5} A_{1}$ & $\frac{1}{25} A_{6}$ & $\frac{1}{25} A_{2}$ \\
\hline$V_{11}$ & $V_{12}$ & $V_{13}$ & $V_{14}$ & $V_{15}$ & $V_{16}$ & $V_{17}$ & $V_{18}$ & $V_{19}$ & $V_{20}$ \\
\hline$-\frac{1}{125} A_{10}$ & 0 & $-\frac{1}{5} A_{6}$ & $\frac{2}{5}\left(-A_{8}+A_{13}-A_{14}\right)$ & $-\frac{1}{5} A_{2}$ & $\frac{1}{25} A_{10}$ & $-\frac{1}{5} A_{6}$ & $-\frac{1}{5} A_{10}$ & $-\frac{1}{5} A_{11}$ & $-\frac{1}{5} A_{12}$ \\
\hline
\end{tabular}

The $b_{6}$ coefficient is

$$
\begin{aligned}
b_{6}(\mathcal{O})= & \frac{1}{(4 \pi)^{3} 7 !}\left[\frac{6}{5} A_{1}+\frac{1}{5} A_{2}-2 A_{3}-4 A_{4}+9 A_{5}-8 A_{7}+\frac{8}{5} A_{8}+12 A_{9}\right. \\
& \left.-\frac{7}{225} A_{10}+\frac{14}{15} A_{11}-\frac{14}{15} A_{12}-\frac{32}{45} A_{13}-\frac{16}{15} A_{14}-\frac{16}{3} A_{15}+\frac{44}{9} A_{16}+\frac{80}{9} A_{17}\right]
\end{aligned}
$$




\section{A.2 Weyl fermion}

The appropriate second order operator for the Dirac fermion may be obtained as the square of the Dirac operator:

$$
\mathcal{O} \psi=-\square \psi+\frac{1}{4} R \psi .
$$

The endomorphism and curvature of the connection coincide with the result obtained in $[16]$.

$$
E=-\frac{1}{4} R, \quad F_{i j}=\frac{1}{4} R_{i j a b} \gamma^{a b} .
$$

Then the $V$-terms contributing to the anomaly are (after tracing):

\begin{tabular}{|c|c|c|c|c|c|c|c|c|c|}
\hline$V_{1}$ & $V_{2}$ & $V_{3}$ & $V_{4}$ & $V_{5}$ & $V_{6}$ & $V_{7}$ & $V_{8}$ & $V_{9}$ & $V_{10}$ \\
\hline$-\frac{1}{2} A_{5}$ & $A_{4}-A_{3}$ & $-\frac{1}{2} A_{9}$ & $\frac{1}{2} A_{17}$ & $-\frac{1}{2} A_{16}$ & $-\frac{1}{2} A_{15}$ & $-\frac{1}{2} A_{12}$ & $-A_{1}$ & $\frac{1}{4} A_{6}$ & $\frac{1}{4} A_{2}$ \\
\hline$V_{11}$ & $V_{12}$ & $V_{13}$ & $V_{14}$ & $V_{15}$ & $V_{16}$ & $V_{17}$ & $V_{18}$ & $V_{19}$ & $V_{20}$ \\
\hline$-\frac{1}{16} A_{10}$ & $\frac{1}{8} A_{12}$ & $-A_{6}$ & $-2\left(A_{8}-A_{13}+A_{14}\right)$ & $-A_{2}$ & $\frac{1}{4} A_{10}$ & $-A_{6}$ & $-A_{10}$ & $-A_{11}$ & $-A_{12}$ \\
\hline
\end{tabular}

The $b_{6}$ coefficient is

$$
\begin{aligned}
b_{6}(\mathcal{O})= & \frac{4}{(4 \pi)^{3} 7 !}\left[-3 A_{1}+\frac{5}{4} A_{2}-9 A_{3}+3 A_{4}-5 A_{5}+\frac{7}{2} A_{6}-8 A_{7}-4 A_{8}-9 A_{9}\right. \\
& \left.-\frac{35}{72} A_{10}+\frac{7}{3} A_{11}+\frac{49}{24} A_{12}+\frac{44}{9} A_{13}-\frac{20}{3} A_{14}+\frac{5}{3} A_{15}-\frac{101}{18} A_{16}-\frac{109}{9} A_{17}\right] .
\end{aligned}
$$

\section{A.3 Vector}

The $(0,1,0)$ vector representation of $\mathrm{SU}(4)$ is a one form, so the correct Laplacian may be obtained by computing the Hodge-deRham operator $d \delta+\delta d$. We get

$$
\mathcal{O} A_{\mu}=-\square A_{\mu}+R_{\mu}^{\nu} A_{\nu} .
$$

The endomorphism and curvature of the connection here are:

$$
E_{b}^{a}=-R_{b}^{a}, \quad\left(F_{i j}\right)_{b}^{a}=R_{b i j}^{a},
$$

so that (after tracing)

\begin{tabular}{|c|c|c|c|c|c|c|c|c|c|}
\hline$V_{1}$ & $V_{2}$ & $V_{3}$ & $V_{4}$ & $V_{5}$ & $V_{6}$ & $V_{7}$ & $V_{8}$ & $V_{9}$ & $V_{10}$ \\
\hline$-A_{5}$ & $2\left(A_{4}-A_{3}\right)$ & $-A_{9}$ & $A_{17}$ & $-A_{16}$ & $-A_{15}$ & $-A_{12}$ & $-A_{1}$ & $A_{7}$ & $A_{3}$ \\
\hline$V_{11}$ & $V_{12}$ & $V_{13}$ & $V_{14}$ & $V_{15}$ & $V_{16}$ & $V_{17}$ & $V_{18}$ & $V_{19}$ & $V_{20}$ \\
\hline$-A_{13}$ & $A_{15}$ & $-A_{6}$ & $-2\left(A_{8}-A_{13}+A_{14}\right)$ & $-A_{2}$ & $A_{11}$ & $-A_{6}$ & $-A_{10}$ & $-A_{11}$ & $-A_{12}$ \\
\hline
\end{tabular}

and

$$
\begin{aligned}
b_{6}(\mathcal{O})= & \frac{1}{(4 \pi)^{3} 7 !}\left[24 A_{1}-66 A_{2}+3529 A_{3}+32 A_{4}-58 A_{5}-140 A_{6}+792 A_{7}-32 A_{8}-96 A_{9}\right. \\
& \left.-\frac{140}{3} A_{10}+420 A_{11}-70 A_{12}-\frac{2600}{3} A_{13}+16 A_{14}+444 A_{15}-\frac{164}{3} A_{16}-\frac{344}{3} A_{17}\right] .
\end{aligned}
$$




\section{A.4 Self-dual three-form}

The field which transforms under the $(2,0,0)$ representation is the 10 -component self-dual three-form. A three-index antisymmetric tensor has 20 components and the self-duality condition removes half of these. The operator acting on this field is

$$
\begin{aligned}
\mathcal{O} C_{\mu \nu \rho}= & -\square C_{\mu \nu \rho}+R_{\mu}{ }^{\lambda} C_{\lambda \nu \rho}+R_{\nu}{ }^{\lambda} C_{\mu \lambda \rho}+R_{\rho}{ }^{\lambda} C_{\mu \nu \lambda} \\
& -R_{\mu \nu}{ }^{\lambda \sigma} C_{\lambda \sigma \rho}-R_{\nu \rho}{ }^{\lambda \sigma} C_{\mu \lambda \sigma}-R_{\rho \mu}{ }^{\lambda \sigma} C_{\lambda \nu \sigma} .
\end{aligned}
$$

This means that the endomorphism and connection curvature are given by

$$
E_{a b c}^{d e f}=-3 R_{[a}^{[d} \delta_{b}^{e} \delta_{c]}^{f]}+3 R_{[a b}^{[d e} \delta_{c]}^{f]}, \quad\left(F_{i j}\right)_{a b c}{ }^{d e f}=-3 R_{i j[a}\left[d \delta_{b}^{e} \delta_{c]}^{f]} .\right.
$$

Then we can compute the relevant terms:

\begin{tabular}{|c|c|c|c|c|}
\hline$V_{1}$ & $V_{2}$ & $V_{3}$ & $V_{4}$ & $V_{5}$ \\
\hline$-6 A_{5}$ & $12\left(A_{4}-A_{3}\right)$ & $-6 A_{9}$ & $6 A_{17}$ & $-6 A_{16}$ \\
\hline$V_{6}$ & $V_{7}$ & $V_{8}$ & $V_{9}$ & $V_{10}$ \\
\hline$-6 A_{15}$ & $-6 A_{12}$ & $-6 A_{1}$ & $2 A_{6}-2 A_{7}+2 A_{9}$ & $2 A_{2}-2 A_{3}+2 A_{5}$ \\
\hline$V_{11}$ & $V_{12}$ & $V_{13}$ & $V_{14}$ & $V_{15}$ \\
\hline$*$ & $2 A_{12}-2 A_{15}+2 A_{16}$ & $-6 A_{6}$ & $-12\left(A_{8}-A_{13}+A_{14}\right)$ & $-6 A_{2}$ \\
\hline$V_{16}$ & $V_{17}$ & $V_{18}$ & $V_{19}$ & $V_{20}$ \\
\hline $2\left(A_{10}-A_{11}+A_{12}\right)$ & $-6 A_{6}$ & $-6 A_{10}$ & $-6 A_{11}$ & $-6 A_{12}$ \\
\hline
\end{tabular}

where $V_{11}=-A_{10}+6 A_{11}-3 A_{12}-6 A_{13}-12 A_{14}+12 A_{15}-2 A_{16}+8 A_{17}$. (Again, all $V$-terms are given after tracing over the representation.) So the $b_{6}$ coefficient is given by

$$
\begin{aligned}
b_{6}(\mathcal{O})=\frac{1}{(4 \pi)^{3} 7 !}[ & -144 A_{1}+172 A_{2}-1216 A_{3}+256 A_{4}+348 A_{5}+392 A_{6}-1840 A_{7} \\
& -192 A_{8}+912 A_{9}+\frac{3080}{9} A_{10}+\frac{12824}{3} A_{11}-\frac{4004}{3} A_{12}-\frac{43472}{9} A_{13} \\
& \left.-\frac{30976}{3} A_{14}+\frac{28408}{3} A_{15}-\frac{11216}{9} A_{16}+\frac{53008}{9} A_{17}\right] .
\end{aligned}
$$

The self-duality condition reduces each of these terms by a factor of two, reproducing the $A_{16}$ and $A_{17}$ terms found in table 1.

\section{A.5 Gravitino}

The gravitino with the gauge condition $\gamma^{\mu} \psi_{\mu}=0$ corresponds to the $(1,1,0)$ representation. In this case the operator $\mathcal{O}$ is the square of the Rarita-Schwinger operator:

$$
\mathcal{O} \psi_{\mu}=-\square \psi_{\mu}+\frac{1}{4} R \psi_{\mu}-\frac{1}{2} \gamma^{\rho} \gamma^{\sigma} R_{\rho \sigma \mu \nu} \psi^{\nu}
$$


The endomorphism and connection curvature are given by

$$
E_{b}{ }^{a}=-\frac{1}{4} R \delta_{b}^{a}+\frac{1}{2} R_{c d b}{ }^{a} \gamma^{c d}, \quad\left(F_{i j}\right)_{b}{ }^{a}=\frac{1}{4} R_{i j c d} \gamma^{c d} \delta_{b}^{a}+R_{i j b}{ }^{a},
$$

so (after tracing)

\begin{tabular}{|c|c|c|c|c|}
\hline$V_{1}$ & $V_{2}$ & $V_{3}$ & $V_{4}$ & $V_{5}$ \\
\hline$-7 A_{15}$ & $-7 A_{12}$ & $-6 A_{1}$ & $\frac{3}{2} A_{6}+2 A_{9}$ & $\frac{3}{2} A_{2}+2 A_{5}$ \\
\hline$V_{6}$ & $V_{7}$ & $V_{8}$ & $V_{9}$ & $V_{10}$ \\
\hline$-7 A_{5}$ & $14\left(A_{4}-A_{3}\right)$ & $-7 A_{9}$ & $7 A_{17}$ & $-7 A_{16}$ \\
\hline$V_{11}$ & $V_{12}$ & $V_{13}$ & $V_{14}$ & $V_{15}$ \\
\hline$-\frac{3}{8} A_{10}-\frac{3}{2} A_{12}+4 A_{17}$ & $\frac{7}{4} A_{12}+2 A_{16}$ & $-6 A_{6}$ & $-12\left(A_{8}-A_{13}+A_{14}\right)$ & $-6 A_{2}$ \\
\hline$V_{16}$ & $V_{17}$ & $V_{18}$ & $V_{19}$ & $V_{20}$ \\
\hline$\frac{3}{2} A_{10}+2 A_{12}$ & $-6 A_{6}$ & $-6 A_{10}$ & $-6 A_{11}$ & $-6 A_{12}$ \\
\hline
\end{tabular}

and

$$
\begin{aligned}
b_{6}(\mathcal{O})=\frac{1}{(4 \pi)^{3} 7 !}[ & -60 A_{1}+25 A_{2}-404 A_{3}+284 A_{4}+292 A_{5}+70 A_{6}-160 A_{7} \\
& -80 A_{8}+828 A_{9}-\frac{175}{18} A_{10}+\frac{140}{3} A_{11}-\frac{1435}{6} A_{12}-\frac{880}{9} A_{13} \\
& \left.-\frac{400}{3} A_{14}+\frac{772}{3} A_{15}+\frac{3526}{9} A_{16}+\frac{22012}{9} A_{17}\right] .
\end{aligned}
$$

\section{A.6 Two-form}

The adjoint representation $(1,0,1)$ corresponds to the two-form computed in [16].

$$
\mathcal{O} B_{\mu \nu}=-\square B_{\mu \nu}+R_{\mu}^{\lambda} B_{\lambda \nu}-R_{\nu}^{\lambda} B_{\lambda \mu}-R_{\mu \nu}{ }^{\rho \sigma} B_{\rho \sigma} .
$$

This means that the endomorphism and connection curvature are given by

$$
E_{a b}{ }^{c d}=-2 R_{[a}^{[c} \delta_{b]}^{d]}+R_{a b}^{c d}, \quad\left(F_{i j}\right)_{a b}{ }^{c d}=2 R_{i j[a}^{[c} \delta_{b]}^{d]},
$$

so (after tracing)

\begin{tabular}{|c|c|c|c|c|}
\hline$V_{1}$ & $V_{2}$ & $V_{3}$ & $V_{4}$ & $V_{5}$ \\
\hline$-4 A_{5}$ & $8\left(A_{4}-A_{3}\right)$ & $-4 A_{9}$ & $4 A_{17}$ & $-4 A_{16}$ \\
\hline$V_{6}$ & $V_{7}$ & $V_{8}$ & $V_{9}$ & $V_{10}$ \\
\hline$-4 A_{15}$ & $-4 A_{12}$ & $-4 A_{1}$ & $A_{6}+A_{9}$ & $A_{2}+A_{5}$ \\
\hline$V_{11}$ & $V_{12}$ & $V_{13}$ & $V_{14}$ & $V_{15}$ \\
\hline$-3 A_{11}+4 A_{13}+6 A_{14}-6 A_{15}+A_{16}$ & $A_{12}+A_{16}$ & $-4 A_{6}$ & $-8\left(A_{8}-A_{13}+A_{14}\right)$ & $-4 A_{2}$ \\
\hline$V_{16}$ & $V_{17}$ & $V_{18}$ & $V_{19}$ & $V_{20}$ \\
\hline$A_{10}+A_{12}$ & $-4 A_{6}$ & $-4 A_{10}$ & $-4 A_{11}$ & $-4 A_{12}$ \\
\hline
\end{tabular}


and

$$
\begin{aligned}
& b_{6}(\mathcal{O})=\frac{1}{(4 \pi)^{3} 7 !}\left[-66 A_{1}+3 A_{2}-254 A_{3}+164 A_{4}+107 A_{5}+28 A_{6}-120 A_{7}-88 A_{8}+348 A_{9}\right. \\
& \left.+\frac{595}{3} A_{10}-2478 A_{11}+518 A_{12}+\frac{10384}{3} A_{13}-4912 A_{14}-4896 A_{15}+\frac{2992}{3} A_{16}-\frac{1616}{3} A_{17}\right] .
\end{aligned}
$$

\section{A.7 Graviton}

The symmetric spin-two field is the $(0,2,0)$ representation. The appropriate kinetic operator is the Lichnerowicz operator [34]:

$$
\mathcal{O} h_{\mu \nu}=-\square h_{\mu \nu}+R_{\mu}{ }^{\lambda} h_{\lambda \nu}+R_{\nu}{ }^{\lambda} h_{\lambda \mu}-2 R_{\mu \rho \nu \sigma} h^{\rho \sigma} .
$$

The endomorphism and connection are given by

$$
E_{\mu \nu}^{\rho \sigma}=-2 R_{\{\mu}^{\{\rho} \delta_{\nu\}}^{\sigma\}}+R_{\mu}^{\rho}{ }_{\nu}^{\sigma}+R_{\mu}^{\sigma}{ }^{\rho} \rho, \quad\left(F_{a b}\right)_{\mu \nu}^{\rho \sigma}=2 R_{a b\{\mu}^{\{\rho} \delta_{\nu\}}^{\sigma\}} .
$$

Then we can compute the relevant terms:

\begin{tabular}{|c|c|c|c|c|}
\hline$V_{1}$ & $V_{2}$ & $V_{3}$ & $V_{4}$ & $V_{5}$ \\
\hline$-8 A_{5}$ & $16\left(A_{4}-A_{3}\right)$ & $-8 A_{9}$ & $8 A_{17}$ & $-8 A_{16}$ \\
\hline$V_{6}$ & $V_{7}$ & $V_{8}$ & $V_{9}$ & $V_{10}$ \\
\hline$-8 A_{15}$ & $-8 A_{12}$ & $-8 A_{1}$ & $A_{6}+12 A_{7}+3 A_{9}$ & $A_{2}+12 A_{3}+3 A_{5}$ \\
\hline$V_{11}$ & $V_{12}$ & $V_{13}$ & $V_{14}$ & $V_{15}$ \\
\hline$*$ & $A_{12}+12 A_{15}+3 A_{16}$ & $-8 A_{6}$ & $-16\left(A_{8}-A_{13}+A_{14}\right)$ & $-8 A_{2}$ \\
\hline$V_{16}$ & $V_{17}$ & $V_{18}$ & $V_{19}$ & $V_{20}$ \\
\hline$A_{10}+12 A_{11}+3 A_{12}$ & $-8 A_{6}$ & $-8 A_{10}$ & $-8 A_{11}$ & $-8 A_{12}$ \\
\hline
\end{tabular}

where $V_{11}=-3 A_{11}-16 A_{13}-6 A_{14}-18 A_{15}-A_{16}+8 A_{17}$, and these terms are given after tracing over the representation. The $b_{6}$ coefficient is

$$
\begin{aligned}
b_{6}(\mathcal{O})=\frac{1}{(4 \pi)^{3} 7 !}[ & -312 A_{1}-584 A_{2}-4552 A_{3}+368 A_{4}+544 A_{5}-1064 A_{6}+9920 A_{7} \\
& -416 A_{8}+1416 A_{9}-\frac{560}{9} A_{10}+\frac{7952}{3} A_{11}+\frac{2968}{3} A_{12}-\frac{117056}{9} A_{13} \\
& \left.-\frac{16528}{3} A_{14}-\frac{29216}{3} A_{15}-\frac{1388}{9} A_{16}+\frac{49984}{9} A_{17}\right] .
\end{aligned}
$$

\section{B Heat kernel for general spins}

We are interested in a general formula to compute the heat kernel coefficients for spins higher than two, analogous to the algorithm [23] in four dimensions. We consider fields transforming in an irreducible representation of the spacetime symmetry group that are acted on by a generalized second-order operator $\Delta=-\square-E$. In four dimensions, the 
method of computing the heat kernels for general representations assumes that the endomorphism term $E$ for fields transforming as $(A, B)$ of $\mathrm{SO}(4) \simeq \mathrm{SU}(2)_{L} \times \mathrm{SU}(2)_{R}$ is given by:

$$
E=\Sigma_{a b} R^{a b c d} \Sigma_{c d} \quad \text { or } \quad E=\frac{1}{A} \Sigma_{a b} R_{+}^{a b c d} \Sigma_{c d}
$$

for bosonic $(A+B=$ integer $)$ or fermionic $(A+B=$ half-integer, $A>B)$ representations, respectively. Here $R_{+}^{a b c d}=\frac{1}{2}\left(R^{a b c d}+R^{* a b c d}\right)$. This prescription is shown to be valid for fields up to spin two in four dimensions, and is conjectured to be the appropriate operator for general spins. In six dimensions, it appears that this prescription is reasonable for bosonic representations, but straightforward generalizations for fermions fail to reproduce the conventional endomorphism terms for the Weyl fermion and gravitino. So it remains unclear what endomorphism term is appropriate for general fermions. Below we use this method for bosonic representations to compute all the $V$ terms, which are built out of the endomorphism $E$ and the connection $F_{i j}$.

\section{B.1 Tracing over generators}

Computing the heat kernel using this method requires computing the trace of a number of generators; the most we will need is six, as $E^{3} \sim \Sigma^{6}$. We perform these traces using the algorithm presented in [35], which requires expanding the trace into a sum of symmetric traces, and then writing each symmetric trace in a basis of orthogonal tensors and higher order Dynkin indices. For example, the trace of two generators of an irreducible representation is

$$
\operatorname{Tr}\left[T_{R}^{A} T_{R}^{B}\right]=I_{2}(R) g^{A B} .
$$

Here $R$ refers to the representation, and the capital Roman letters $A, B, \ldots=1,2, \ldots, 15$ label the generators of $\mathrm{SU}(4)$. Each $\mathrm{SU}(4)$ index is interchangeable with a pair of antisymmetrized six-dimensional spacetime indices $\{\mu, \nu\}$.

If the number of generators is greater than two, we will first need to break the trace into a sum of symmetrized traces. For a trace of $n$ generators, this is accomplished by writing out each of the $n$ ! terms in the symmetrized trace, and then using commutation relations to return each term to the original order, plus a number of traces of lower numbers of generators. For example, we may look at the trace of six generators. First consider the symmetrized trace

$$
\begin{aligned}
\operatorname{STr} & {\left[T_{A} T_{B} T_{C} T_{D} T_{E} T_{F}\right] } \\
& =\frac{1}{6 !}\left(\operatorname{Tr}\left[T_{A} T_{B} T_{C} T_{D} T_{E} T_{F}\right]+\operatorname{Tr}\left[T_{B} T_{A} T_{C} T_{D} T_{E} T_{F}\right]+718 \text { more terms }\right) .
\end{aligned}
$$

Using the fact that $T_{B} T_{A}=\left[T_{B}, T_{A}\right]+T_{A} T_{B}$ and the algebra, we may rewrite this trace as

$$
\begin{aligned}
& \mathrm{S} \operatorname{Tr}\left[T_{A} T_{B} T_{C} T_{D} T_{E} T_{F}\right]=\frac{1}{6 !}\left(\operatorname{Tr}\left[T_{A} T_{B} T_{C} T_{D} T_{E} T_{F}\right]\right. \\
& \left.\quad+\operatorname{Tr}\left[T_{A} T_{B} T_{C} T_{D} T_{E} T_{F}\right]+\operatorname{Tr}\left[f_{B A X} T^{X} T_{C} T_{D} T_{E} T_{F}\right]+718 \text { more terms }\right) .
\end{aligned}
$$

This gives two factors of the non-symmetrized trace plus a term which has a trace over only five generators. Each of the other 718 terms may be dealt with in the same way: commute 
the generators to put them in the order $(A B C D E F)$ and keep track of all of the traces over five generators which are picked up along the way. This adds $5 \cdot 5$ ! terms with five generators. Using this and rearranging the trace and symmetric trace, we get the schematic relation

$$
\operatorname{Tr}\left[T_{A} T_{B} T_{C} T_{D} T_{E} T_{F}\right]=\operatorname{S} \operatorname{Tr}\left[T_{A} T_{B} T_{C} T_{D} T_{E} T_{F}\right]-\frac{1}{6 !} \cdot 600 \operatorname{Tr}[T T T T T] .
$$

Each of these five-generator traces may be treated the same way- they each yield a symmetric trace with five generators plus $4 \cdot 4$ ! terms with a trace over four generators. Schematically, the trace may be expanded as

$$
\begin{aligned}
& \operatorname{Tr}\left[T_{A} T_{B} T_{C} T_{D} T_{E} T_{F}\right] \\
& \quad=\operatorname{STr}\left[T_{A} T_{B} T_{C} T_{D} T_{E} T_{F}\right]-\frac{1}{6 !}\left(600\left(\operatorname{STr}[T T T T T]-\frac{1}{5 !} \cdot 96 \operatorname{Tr}[T T T T]\right)\right),
\end{aligned}
$$

and so on, until the result is a sum of symmetric traces of $2,3,4,5$, and 6 generators. Clearly this computation is not tractable by hand. Using the XACT package for Mathematica, we calculated all the necessary terms. The symmetric traces over an odd number of generators cancel each other out (which appears to be a sort of generalization of Furry's theorem). The result of this procedure includes a symmetric trace over six generators and a large number of symmetric traces over four generators and two generators.

\section{B.2 Orthogonal tensors}

The symmetrized traces may be expanded in a set of orthogonal symmetric tensors. The two needed for this calculation are

$$
\mathrm{S} \operatorname{Tr}\left[T^{A} T^{B} T^{C} T^{D}\right]=I_{4}(R) d_{\perp}^{A B C D}+I_{2,2}(R)\left(\delta^{A B} \delta^{C D}+\delta^{A C} \delta^{B D}+\delta^{A D} \delta^{B C}\right) / 3,
$$

and

$$
\begin{aligned}
\mathrm{S} \operatorname{Tr} & {\left[T^{A} T^{B} T^{C} T^{D} T^{E} T^{F}\right]=I_{6}(R) d_{\perp}^{A B C D E F}+I_{4,2}(R)\left(d_{\perp}^{A B C D} \delta^{E F}+d_{\perp}^{A B C E} \delta^{D F}+\cdots\right) / 15 } \\
& +I_{3,3}(R)\left(d_{\perp}^{A B C} d_{\perp}^{D E F}+d_{\perp}^{A B D} d_{\perp}^{C E F}+\cdots\right) / 10+I_{2,2,2}(R)\left(\delta^{A B} \delta^{C D} \delta^{E F}+\cdots\right) / 15 .
\end{aligned}
$$

Note that $I_{6}=0$ for all representations of $\mathrm{SU}(4)$. The tensors $d_{\perp}^{A B C D}$ and $d_{\perp}^{A B C}$ are fixed by the condition of orthogonality; $d_{\perp}^{A B C}$ is the six-dimensional epsilon tensor (recalling that $A=\left\{\mu_{1} \nu_{1}\right\}$, etc.) The fourth order $d_{\perp}^{A B C D}$ may be expressed in terms of the six-dimensional metric - its terms include $g^{\mu_{1} \nu_{4}} g^{\mu_{2} \nu_{3}} g^{\mu_{3} \nu_{2}} g^{\mu_{4} \nu_{1}}$ and the other 47 ways of arranging the indices. The indices $I_{4,2}, I_{3,3}$, and $I_{2,2,2}$ are not unique; imposing orthogonality and other group-theoretic relations yields the system of equations (158)-(160) in [35]. Solving these allows $I_{4,2}, I_{3,3}$, and $I_{2,2,2}$ to be expressed in terms of the Dynkin indices $I_{4}$, $I_{3}$, and $I_{2}$.

\section{B.3 Dynkin indices}

A representation $R$ with Dynkin labels $(a, b, c)$ has dimension

$$
\operatorname{Dim}_{R}(a, b, c)=\frac{1}{12}(a+1)(b+1)(c+1)(a+b+2)(b+c+2)(a+b+c+3) .
$$


The Weyl character formula may be used to show that

$$
I_{2}(a, b, c)=\frac{\operatorname{Dim}_{R}}{60}\left(3 a^{2}+2 a(2 b+c+6)+4 b^{2}+4 b(c+4)+3 c(c+4)\right) .
$$

The third and fourth order generalization to this index were computed in [36], which finds

$$
\begin{aligned}
I_{3}(a, b, c)= & \frac{\operatorname{Dim}_{R}}{120}(a-c)(a+c+2)(a+2 b+c+4) \\
I_{4}(a, b, c)= & \frac{\operatorname{Dim}_{R}}{3360}\left(3 a^{4}+8 a^{3} b+4 a^{3} c+24 a^{3}+2 a^{2} b^{2}+2 a^{2} b c+30 a^{2} b\right. \\
& -4 a^{2} c^{2}+6 a^{2} c+54 a^{2}-12 a b^{3}-18 a b^{2} c-50 a b^{2}+2 a b c^{2}-28 a b c \\
& -34 a b+4 a c^{3}+6 a c^{2}-2 a c+24 a-6 b^{4}-12 b^{3} c-48 b^{3}+2 b^{2} c^{2} \\
& \left.-50 b^{2} c-122 b^{2}+8 b c^{3}+30 b c^{2}-34 b c-104 b+3 c^{4}+24 c^{3}+54 c^{2}+24 c\right) .
\end{aligned}
$$

\section{B.4 Results}

As the trace of each of the $V_{a}$ coefficients may be reduced to a trace of generators variously contracted with the Riemann tensor, this method will allow each of them to be computed. The entire list of traced coefficients is presented here:

$$
\begin{aligned}
V_{1} & =-\frac{A_{5}}{2} I_{2}, \quad V_{2}=\left(A_{4}-A_{3}\right) I_{2}, \quad V_{3}=-\frac{A_{9}}{2} I_{2}, \quad V_{4}=\frac{A_{17}}{2} I_{2} \\
V_{5} & =-\frac{A_{16}}{2} I_{2}, \quad V_{6}=-\frac{A_{15}}{2} I_{2}, \quad V_{7}=-\frac{A_{12}}{2} I_{2}, \quad V_{8}=-\frac{A_{1}}{2} I_{2}, \\
V_{9} & =\left(-\frac{A_{6}}{51}+\frac{A_{7}}{6}-\frac{25 A_{9}}{204}\right) I_{2}+\left(\frac{15 A_{6}}{68}+\frac{15 A_{9}}{34}\right) \frac{I_{2}^{2}}{\operatorname{Dim}_{R}}+\left(\frac{11 A_{6}}{51}-\frac{4 A_{7}}{3}+\frac{5 A_{9}}{51}\right) I_{4}, \\
V_{10} & =\left(-\frac{A_{2}}{51}+\frac{A_{3}}{6}-\frac{25 A_{5}}{204}\right) I_{2}+\left(\frac{15 A_{2}}{68}+\frac{15 A_{5}}{34}\right) \frac{I_{2}^{2}}{\operatorname{Dim}_{R}}+\left(\frac{11 A_{2}}{51}-\frac{4 A_{3}}{3}+\frac{5 A_{5}}{51}\right) I_{4}, \\
V_{11} & =\left(\frac{A_{10}}{612}-\frac{11 A_{11}}{357}-\frac{3 A_{12}}{238}-\frac{55 A_{13}}{2142}+\frac{151 A_{14}}{714}+\frac{3 A_{15}}{34}-\frac{383 A_{16}}{4284}-\frac{338 A_{17}}{1071}\right) I_{2} \\
& +\left(\frac{5 A_{10}}{136}-\frac{375 A_{11}}{952}+\frac{1095 A_{12}}{3808}+\frac{115 A_{13}}{476}+\frac{345 A_{14}}{952}-\frac{165 A_{15}}{136}+\frac{325 A_{16}}{476}+\frac{725 A_{17}}{952}\right) \frac{I_{2}^{2}}{\operatorname{Dim}_{R}} \\
& +\left(\frac{10 A_{10}}{153}-\frac{41 A_{11}}{51}+\frac{6 A_{12}}{17}+\frac{280 A_{13}}{153}+\frac{38 A_{14}}{51}-\frac{42 A_{15}}{17}+\frac{43 A_{16}}{153}-\frac{8 A_{17}}{153}\right) I_{4} \\
& +\left(-\frac{5 A_{10}}{68}-\frac{165 A_{11}}{952}-\frac{1845 A_{12}}{3808}+\frac{115 A_{13}}{476}+\frac{345 A_{14}}{952}-\frac{45 A_{15}}{136}-\frac{305 A_{16}}{476}-\frac{115 A_{17}}{952}\right) \frac{I_{2}^{3}}{\operatorname{Dim}_{R}^{2}} \\
& +\left(-\frac{7 A_{10}}{24}+\frac{209 A_{11}}{56}-\frac{183 A_{12}}{224}-\frac{437 A_{13}}{84}-\frac{437 A_{14}}{56}+\frac{57 A_{15}}{8}-\frac{101 A_{16}}{84}+\frac{437 A_{17}}{168}\right) \frac{I_{3}^{2}}{\operatorname{Dim}_{R}} \\
& +\left(-\frac{13 A_{10}}{102}+\frac{4 A_{11}}{17}-\frac{12 A_{12}}{17}+\frac{76 A_{13}}{51}+\frac{38 A_{14}}{17}+\frac{54 A_{15}}{17}-\frac{2 A_{16}}{51}-\frac{38 A_{17}}{51}\right) \frac{I_{2} I_{4}}{\operatorname{Dim}_{R}}, \\
V_{12} & =\left(-\frac{A_{12}}{51}+\frac{A_{15}}{6}-\frac{25 A_{16}}{204}\right) I_{2}+\left(\frac{15 A_{12}}{68}+\frac{15 A_{16}}{34}\right) \frac{I_{2}^{2}}{\operatorname{Dim}_{R}}+\left(\frac{11 A_{12}}{51}-\frac{4 A_{15}}{3}+\frac{5 A_{16}}{51}\right) I_{4}, \\
V_{13} & =-\frac{A_{6}}{2} I_{2}, \quad V_{14}=-\left(A_{8}-A_{13}+A_{14}\right) I_{2}, \quad-\frac{A_{2}}{2} I_{2},
\end{aligned}
$$




$$
\begin{aligned}
& V_{16}=\left(-\frac{A_{10}}{51}+\frac{A_{11}}{6}-\frac{25 A_{12}}{204}\right) I_{2}+\left(\frac{15 A_{10}}{68}+\frac{15 A_{12}}{34}\right) \frac{I_{2}^{2}}{\operatorname{Dim}_{R}}+\left(\frac{11 A_{10}}{51}-\frac{4 A_{11}}{3}+\frac{5 A_{12}}{51}\right) I_{4}, \\
& V_{17}=-\frac{A_{6}}{2} I_{2}, \quad V_{18}=-\frac{A_{10}}{2} I_{2}, \quad V_{19}=-\frac{A_{11}}{2} I_{2}, \quad V_{20}=-\frac{A_{12}}{2} I_{2} .
\end{aligned}
$$

Since these expressions pertain to an endomorphism of the form $E=\Sigma_{a b} R^{a b c d} \Sigma_{c d}$, where $\Sigma_{a b}$ are SU(4) generators in an arbitrary representation specified by Dynkin labels $(a, b, c)$, we refer to this as the "group theory method" for determining the heat kernel coefficients.

Now that the $V_{a}$ 's are known, we may compute the $b_{6}$ coefficient using the group theory method. We present the coefficient for a representation $R$ on Ricci-flat backgrounds:

$$
\begin{aligned}
&\left.b_{6}(R)\right|_{R_{a b}=0}=\frac{1}{(4 \pi)^{3} 7 !}[ A_{5}\left(\frac{3150 I_{2}^{2}}{17 \operatorname{Dim}_{R}}+9 \operatorname{Dim}_{R}-\frac{1827 I_{2}}{17}+\frac{700 I_{4}}{17}\right) \\
&+A_{9}\left(\frac{6300 I_{2}^{2}}{17 \operatorname{Dim}_{R}}+12 \operatorname{Dim}_{R}-\frac{3178 I_{2}}{17}+\frac{1400 I_{4}}{17}\right) \\
&+A_{16}\left(-\frac{9150 I_{2}^{3}}{17 \operatorname{Dim}_{R}^{2}}+\frac{12900 I_{2}^{2}}{17 \operatorname{Dim}_{R}}-\frac{560 I_{2} I_{4}}{17 \operatorname{Dim}_{R}}-\frac{1010 I_{3}^{2}}{\operatorname{Dim}_{R}}+\frac{44 \operatorname{Dim}_{R}}{9}-\frac{8597 I_{2}}{51}+\frac{14140 I_{4}}{51}\right) \\
&\left.+A_{17}\left(-\frac{1725 I_{2}^{3}}{17 \operatorname{Dim}_{R}^{2}}+\frac{10875 I_{2}^{2}}{17 \operatorname{Dim}_{R}}-\frac{10640 I_{2} I_{4}}{17 \operatorname{Dim}_{R}}+\frac{2185 I_{3}^{2}}{\operatorname{Dim}_{R}}+\frac{80 \operatorname{Dim}_{R}}{9}-\frac{17804 I_{2}}{51}-\frac{2240 I_{4}}{51}\right)\right] .
\end{aligned}
$$

In general, the full $b_{6}$ coefficients obtained by the group theory method do not match the expressions (A.6) and (A.15), for the fermion and gravitino, respectively, as the group theory method does not correspond to the square of the Dirac operator when acting on fermions. This indicates that some modification may be necessary for fermionic representations, as was already noted in the four-dimensional case [23]. Curiously, however, this mismatch disappears when restricted to Ricci-flat backgrounds. This suggests that (B.13) may potentially be valid for fermions as well as bosons. If this were true, we could then derive a general expression for $\delta(c-a)$ for arbitrary higher spin supermultiplets.

Finally, we find that the expression $\delta \mathcal{A}$ in (2.5) vanishes on arbitrary (ie not just Ricciflat) backgrounds for long multiplets using the group theory method for the heat kernel. This is in contrast to the conventional method where the fermions are treated by squaring the Dirac operator. There, $\delta \mathcal{A}$ for long multiplets only vanished on Ricci-flat backgrounds, but was otherwise non-vanishing on more general backgrounds. This complete vanishing of $\delta \mathcal{A}$ for long multiplets is consistent with expectations from $\mathrm{AdS}_{5} / \mathrm{CFT}_{4}[9,12]$, and lends credibility to the idea that the group theory method may yield the correct expression for $\delta \mathcal{A}$ for general spins.

Open Access. This article is distributed under the terms of the Creative Commons Attribution License (CC-BY 4.0), which permits any use, distribution and reproduction in any medium, provided the original author(s) and source are credited.

\section{References}

[1] J.L. Cardy, Operator content of two-dimensional conformally invariant theories, Nucl. Phys. B 270 (1986) 186 [inSPIRE]. 
[2] A.B. Zamolodchikov, Irreversibility of the flux of the renormalization group in a $2 D$ field theory, JETP Lett. 43 (1986) 730 [Pisma Zh. Eksp. Teor. Fiz. 43 (1986) 565] [INSPIRE].

[3] M. Henningson and K. Skenderis, The holographic Weyl anomaly, JHEP 07 (1998) 023 [hep-th/9806087] [INSPIRE].

[4] A. Bilal and C.-S. Chu, A note on the chiral anomaly in the AdS/CFT correspondence and $1 / N^{2}$ correction, Nucl. Phys. B 562 (1999) 181 [hep-th/9907106] [INSPIRE].

[5] P. Mansfield and D. Nolland, One loop conformal anomalies from AdS/CFT in the Schrödinger representation, JHEP 07 (1999) 028 [hep-th/9906054] [INSPIRE].

[6] P. Mansfield and D. Nolland, Order $1 / N^{2}$ test of the Maldacena conjecture: cancellation of the one loop Weyl anomaly, Phys. Lett. B 495 (2000) 435 [hep-th/0005224] [INSPIRE].

[7] P. Mansfield, D. Nolland and T. Ueno, Order $1 / N^{2}$ test of the Maldacena conjecture. 2. The full bulk one loop contribution to the boundary Weyl anomaly, Phys. Lett. B 565 (2003) 207 [hep-th/0208135] [INSPIRE].

[8] P. Mansfield, D. Nolland and T. Ueno, The boundary Weyl anomaly in the $N=4 S Y M / t y p e$ IIB supergravity correspondence, JHEP 01 (2004) 013 [hep-th/0311021] [INSPIRE].

[9] A. Arabi Ardehali, J.T. Liu and P. Szepietowski, The spectrum of IIB supergravity on $A d S_{5} \times S^{5} / Z_{3}$ and a $1 / N^{2}$ test of AdS/CFT, JHEP 06 (2013) 024 [arXiv:1304.1540] [INSPIRE].

[10] A. Arabi Ardehali, J.T. Liu and P. Szepietowski, $1 / N^{2}$ corrections to the holographic Weyl anomaly, JHEP 01 (2014) 002 [arXiv:1310.2611] [INSPIRE].

[11] A. Arabi Ardehali, J.T. Liu and P. Szepietowski, The shortened KK spectrum of IIB supergravity on $Y^{p, q}$, JHEP 02 (2014) 064 [arXiv: 1311.4550] [INSPIRE].

[12] M. Beccaria and A.A. Tseytlin, Higher spins in AdS $S_{5}$ at one loop: vacuum energy, boundary conformal anomalies and AdS/CFT, JHEP 11 (2014) 114 [arXiv:1410.3273] [INSPIRE].

[13] A. Arabi Ardehali, J.T. Liu and P. Szepietowski, $c-a$ from the $N=1$ superconformal index, JHEP 12 (2014) 145 [arXiv: 1407.6024] [INSPIRE].

[14] A. Arabi Ardehali, J.T. Liu and P. Szepietowski, Central charges from the $N=1$ superconformal index, Phys. Rev. Lett. 114 (2015) 091603 [arXiv:1411.5028] [INSPIRE].

[15] L. Di Pietro and Z. Komargodski, Cardy formulae for SUSY theories in $d=4$ and $d=6$, JHEP 12 (2014) 031 [arXiv:1407.6061] [INSPIRE].

[16] F. Bastianelli, S. Frolov and A.A. Tseytlin, Conformal anomaly of $(2,0)$ tensor multiplet in six-dimensions and AdS/CFT correspondence, JHEP 02 (2000) 013 [hep-th/0001041] [INSPIRE].

[17] A.A. Tseytlin, $R^{4}$ terms in 11 dimensions and conformal anomaly of $(2,0)$ theory, Nucl. Phys. B 584 (2000) 233 [hep-th/0005072] [InSPIRE].

[18] P. Mansfield, D. Nolland and T. Ueno, Order $1 / N^{3}$ corrections to the conformal anomaly of the $(2,0)$ theory in six-dimensions, Phys. Lett. B 566 (2003) 157 [hep-th/0305015] [INSPIRE].

[19] M. Beccaria, G. Macorini and A.A. Tseytlin, Supergravity one-loop corrections on $A d S_{7}$ and $A d S_{3}$, higher spins and AdS/CFT, Nucl. Phys. B 892 (2015) 211 [arXiv:1412.0489] [INSPIRE]. 
[20] M. Beccaria and A.A. Tseytlin, $C_{T}$ for higher derivative conformal fields and anomalies of $(1,0)$ superconformal $6 d$ theories, JHEP 06 (2017) 002 [arXiv: 1705.00305] [INSPIRE].

[21] M. Beccaria and A.A. Tseytlin, $C_{T}$ for conformal higher spin fields from partition function on conically deformed sphere, JHEP 09 (2017) 123 [arXiv: 1707.02456] [INSPIRE].

[22] S.M. Christensen and M.J. Duff, Axial and conformal anomalies for arbitrary spin in gravity and supergravity, Phys. Lett. B 76 (1978) 571 [INSPIRE].

[23] S.M. Christensen and M.J. Duff, New gravitational index theorems and supertheorems, Nucl. Phys. B 154 (1979) 301 [INSPIRE].

[24] P.B. Gilkey, The spectral geometry of a Riemannian manifold, J. Diff. Geom. 10 (1975) 601 [INSPIRE].

[25] T. Parker and S. Rosenberg, Invariants of conformal laplacians, J. Diff. Geom. 25 (1987) 199.

[26] S. Minwalla, Restrictions imposed by superconformal invariance on quantum field theories, Adv. Theor. Math. Phys. 2 (1998) 783 [hep-th/9712074] [INSPIRE].

[27] V.K. Dobrev, Positive energy unitary irreducible representations of $D=6$ conformal supersymmetry, J. Phys. A 35 (2002) 7079 [hep-th/0201076] [INSPIRE].

[28] J. Bhattacharya, S. Bhattacharyya, S. Minwalla and S. Raju, Indices for superconformal field theories in 3, 5 and 6 dimensions, JHEP 02 (2008) 064 [arXiv:0801.1435] [INSPIRE].

[29] M. Buican, J. Hayling and C. Papageorgakis, Aspects of superconformal multiplets in D $>4$, JHEP 11 (2016) 091 [arXiv:1606.00810] [INSPIRE].

[30] C. Cordova, T.T. Dumitrescu and K. Intriligator, Multiplets of superconformal symmetry in diverse dimensions, arXiv:1612.00809 [INSPIRE].

[31] C. Cordova, T.T. Dumitrescu and K. Intriligator, Anomalies, renormalization group flows and the a-theorem in six-dimensional $(1,0)$ theories, JHEP 10 (2016) 080 [arXiv: 1506.03807] [INSPIRE].

[32] M. Beccaria and A.A. Tseytlin, Conformal anomaly c-coefficients of superconformal $6 d$ theories, JHEP 01 (2016) 001 [arXiv: 1510.02685] [INSPIRE].

[33] S. Yankielowicz and Y. Zhou, Supersymmetric Rényi entropy and anomalies in $6 d(1,0)$ SCFTs, JHEP 04 (2017) 128 [arXiv: 1702.03518] [INSPIRE].

[34] A. Lichnerowicz and C. Møller, Propagators and commutators in general relativity, Proc. Roy. Soc. Lond. A 270 (1962) 342.

[35] T. van Ritbergen, A.N. Schellekens and J.A.M. Vermaseren, Group theory factors for Feynman diagrams, Int. J. Mod. Phys. A 14 (1999) 41 [hep-ph/9802376] [INSPIRE].

[36] S. Okubo, Modified fourth order Casimir invariants and indices for simple Lie algebras, J. Math. Phys. 23 (1982) 8 [INSPIRE]. 